\title{
Protée
}

\section{Le temps photographique de Pierre Perrault}

\section{L'ombre du corps absent}

\section{Stéphane Inkel}

Volume 37, numéro 1, printemps 2009

Corps photographiques / Corps politiques

URI : https://id.erudit.org/iderudit/001356ar

DOI : https://doi.org/10.7202/001356ar

Aller au sommaire du numéro

\section{Éditeur(s)}

Département des arts et lettres - Université du Québec à Chicoutimi

ISSN

0300-3523 (imprimé)

1708-2307 (numérique)

Découvrir la revue

Citer cet article

Inkel, S. (2009). Le temps photographique de Pierre Perrault : l'ombre du corps absent. Protée, 37(1), 67-75. https://doi.org/10.7202/001356ar
Résumé de l'article

Il s'agira dans cet article de confronter les photographies de Pierre Perrault, dans Le Mal du Nord, à l'évolution de l'image cinématographique du corps. Il sera ainsi possible de faire de l'iceberg l'un des quatre objets privilégiés de l'oeuvre, après le corps de la tradition capté au moyen de la chasse au marsouin, la bête lumineuse qui est surtout attente du sujet et l'oumigmag comme corps métaphorique d'une survivance anachronique. Car, si ces photographies du Nord se caractérisent surtout par une absence de l'homme qui reste à interroger, il s'agira de montrer comment les conditions discursives dans lesquelles elles prennent place permettent à Perrault de figurer ce qu'il en est de l'historicité proprement politique du sujet québécois. 


\section{LE TEMPS PHOTOGRAPHIQUE DE PIERRE PERRAULT L'OMBRE DU CORPS ABSENT}

STÉPHANE INKEL

Par-delà la portée directement politique que peut avoir une photographie du corps, ne serait-ce qu'à titre de témoignage, il n'est peut-être pas superflu d'interroger la capture, elle aussi immédiatement politique, propre à toute photographie analysée sous l'angle du dispositif. En effet, le sujet est politique parce qu'il est pris à l'intérieur de toute une série de dispositifs, de "processus objectifs de subjectivations qui le constituent» (Agamben, 2005: 78) et l'inscrivent dans une histoire particulière et une filiation symbolique ${ }^{1}$. Ces dispositifs sont multiples et le plus souvent banals, imperceptibles, loin des excès de la prison comme «dressage des corps» qui a permis à Foucault (1975) de mettre l'accent sur la biopolitique contemporaine. La photographie, en tant qu'elle conditionne les corps à épouser leur propre image, est en soi un dispositif. La conscience de faire partie de l'histoire, qui plus est d'une histoire, nationale ou autre, est également un dispositif puisqu'elle constitue le "régime», chaque fois singulier, à l'intérieur duquel le sujet expérimente son rapport au temps, ses différents va-et-vient entre présent et passé qui constituent le tout de sa mémoire - régime "qui organise ces expériences et permet de les dire» (Hartog, 2003: 27). Le cinéma, en tant que mise en mouvement de l'image photographique et support d'une durée qui est produite par l'expérience du temps engendrée par un «régime d'historicité», est le dispositif qui permet de lier ceux de la photographie et de l'histoire. Il y a donc une puissance politique du cinéma qui peut être utilisée dans un sens ou dans l'autre, c'est-à-dire dans le sens du dispositif ou contre lui, pour le soustraire à la manipulation spectaculaire de l'image dont il est issu.

On sait comment la photographie est l'autre de Pierre Perrault, le dehors d'une pratique qui n'aura croisé le cinéma que par accident, puisqu'elle était avant tout attentive à une parole, existante mais menacée de se perdre, et qui pour l'intellectuel et le poète qu'était Perrault s'avérait déjà perdue. C'est pourtant par son entremise qu'il est possible d'appréhender l'une des ultimes esquisses du rapport à l'histoire présent en filigrane tout au long de l'œuvre. Il existe à cet égard une photographie de Martin Leclerc qui me semble contenir le rapport parfait de Perrault avec l'image cinématographique et photographique. Cette photographie apparaît en page de garde de L'Oumigmatique ou l'objectif documentaire, l'essai tiré de ces deux films «sur l'action de filmer " ${ }^{2}$ que sont L'Oumigmag ou l'objectif documentaire (1993) et Cornouailles (1994). L'on y voit la langue démesurée d'un glacier qui s'avance entre deux sommets 


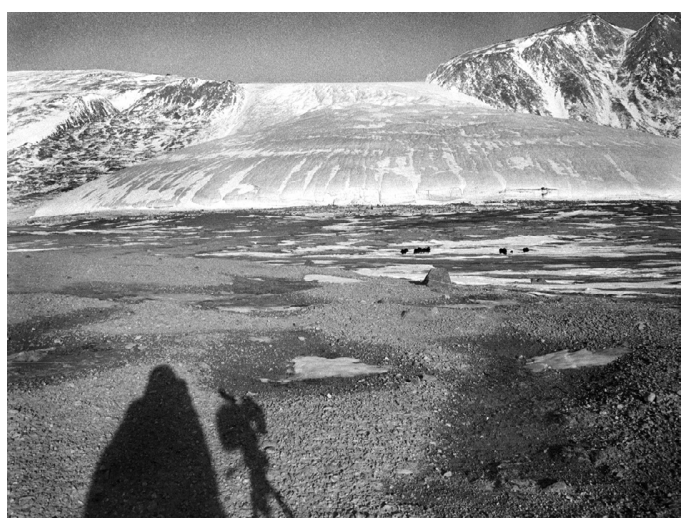

Photographie de M. Leclerc, publiée dans P. Perrault, 1995. Reproduite avec l'autorisation de l'éditeur et du photographe.

rocheux sur le paysage désertique du Nord, au Nouveau-Québec ou sur l'île d'Ellesmere, et un petit troupeau de bœufs musqués au pied du glacier, à une grande distance de l'objectif. À l'avant-plan, on observe l'ombre du photographe et de son appareil de prédilection, la caméra sur son trépied. Du bœuf musqué au sujet de la photographie, il y a un espace saturé par l'absence médiatisée par l'ombre spectrale de ce qui ne peut s'inscrire. Car l'ombre ici est non pas une trace mais bien la préfiguration de ce qui déjà s'avance, traqué par Perrault depuis les premières émissions radiophoniques réalisées à l'Île-aux-Coudres, et qui pourtant ici encore est cantonné hors-champ - appelé, cerné, déjà visible, mais immobilisé par la durée indéfinie du Nord. Quel est le sens de ce corps qui s'absente, dans les derniers films de Perrault? Peut-on soutenir que cette photographie, qui le situe ostensiblement dans un hors-champ contigu à l'image, est le paradigme non seulement de son rapport au cinéma et à l'image, mais aussi du corps politique qui, depuis le début de l'œuvre, constitue son objet privilégié? Corps politique dont la variation continue constituerait en elle-même le sens possible d'une évolution esthétique qui va du primat de la parole au cadre déserté de tout objet des photographies de Perrault publiées en 1999 dans Le Mal du Nord.

Perrault, écrit Gilles Thérien, nous aura conduits [par ses images] à l'invisible, à la frontière entre la réalité des objets et le fantasme épique d'un monde qui ne peut advenir qu'en s'incarnant solidement grâce à ce bestiaire qui dit l'incarnation comme une tentative pour faire naître le pays dans le territoire et l'homme dans la bête [...]. (1999: 174)

Lobjet que je privilégie dans ces pages consiste en une série relativement brève de photographies que Perrault a incluses dans Le Mal du Nord, retranscription de ce qui était à l'origine une émission radiophonique réalisée au cours d'un voyage vers le pôle à bord du brise-glace Pierre-Radisson. Ces photographies ont au moins deux caractéristiques qui peuvent nous intéresser:

1. elles sont de Perrault lui-même, contrairement à celles que l'on trouve dans les versions publiées de ses films, le plus souvent dues au directeur de la photographie, de Michel Brault et Bernard Gosselin à Martin Leclerc;

2. le référent n'est, sauf exception, jamais humain, ni même animal, mais constitué d'icebergs et de banquises, voire de glaces de mer photographiées du haut des airs et dotées d'un haut degré d'abstraction.

Il s'agirait de penser ce que peut vouloir dire cette confrontation obstinée avec ce qui est à la fois sans fin et sans forme, absence de cadre où il serait possible de déposer l'image. Il faut aussi se demander pourquoi Perrault se tourne vers la photographie à la toute fin de son parcours. Car, par-delà la contingence d'une invitation, il faut voir comment Perrault, en transcrivant ses bandes, ne se contente pas d'y ajouter quelques clichés de voyage mais au contraire s'ouvre à la question de l'image, qui dès lors envahit son commentaire. Puisque c'est en définitive l'absence du corps - de tout corps, aussi bien humain qu'animal - qui m'intéresse dans ces photographies, il sera nécessaire de retourner à l'origine de l'œuvre afin d'évaluer le sens de cet objet traqué par Perrault au moyen d'approches aussi différentes que la parole ou la métaphore, l'archivage des gestes de la tradition ou de rites initiatiques qui perdurent dans le présent. Il est possible, à cet égard, d'identifier quatre objets privilégiés dans le parcours cinématographique (et ultimement photographique) de Perrault: le corps de la tradition incarnée par les habitants de l'Île-aux- 
Coudres, notamment dans la reconstitution de la "pêche au marsouin"; la bête lumineuse en tant que proie désirée et attendue d'un rite à préserver; le corps métaphorique de l'oumigmag; enfin les icebergs atemporels des dernières photographies. Il s'agira de voir en quoi ces quatre objets forment autant de métaphores propres à figurer ce qu'il en est du corps politique chez Perrault:

1. corps de la tradition en train de disparaitre;

2. corps messianique du sujet politique à venir;

3. corps du sujet silencieux de la survivance anachronique;

4. absence de corps immobilisée dans une histoire suspendue.

La photographie, on le voit, serait ainsi l'aboutissement d'une démarche de plus en plus silencieuse et immobile, démarche qui recouperait l'évolution de la réflexion politique de Perrault. C'est donc cette intrication politique d'un arrêt sur image en tant qu'ultime visage du devenir qu'il importe de chercher à déplier.

\section{LE CORPS DE LA TRADITION}

On sait comment l'Île-aux-Coudres aura signifié pour Perrault la possibilité de renouer avec une parole vive porteuse de mémoire, cette chouenne, comme il la nommera par mimétisme, que le magnétophone puis l'apparition d'un mode de cinéma, direct ou documentaire, auront permis d'enregistrer, ce qui signifie aussi bien archiver, sauver de l'oubli, que faire exister, littéralement, en créant les conditions nécessaires à la mise en branle du récit ${ }^{3}$. La rencontre de cette parole souveraine, qu'il aura par la suite cherché à «engerber " pour "dire le pays» (Perrault, 1999: 22) en la transposant dans les recueils de poésie Chouennes (1975) et Gélivures (1977), fait office de révélation pour ce jeune intellectuel nourri de littérature étrangère. Aussi cet "émigré de l'intérieur» d'un "pays sans parole», faute de mots pour le dire, se voit-il soudainement confronté à «une parole sans pays» (Perrault, 1995: 21): celle de l'homme populaire qui voit le monde de ses pères en train de s'effriter face à l'avènement de la modernité. On observe comment, dès le début, l'œuvre de Perrault s'avère ainsi dès le début paradoxale: œuvre de mémoire, trouvant dans le direct en train de s'inventer le médium adéquat pour préserver et se réapproprier les gestes d'une présence pérenne, elle ne cesse pourtant de mettre en scène ce qui déjà n'existe plus: chasse au marsouin, construction de "voitures d'eau " désormais inutiles, terres d'Abitibi qui à peine défrichées ne sont bonnes qu'à reboiser pour nourrir les moulins à scies de la région (Un royaume vous attend, 1975). Mais cette tension entre mémoire et perte recoupe elle-même l'une des opérations essentielles du travail de Perrault, celle de la division. En effet, Perrault crée avec ce choix de l'Île-aux-Coudres une forme de catégorisation parmi les divers sujets politiques du Québec des années 1960. On retrouvera ce qu'on pourrait appeler le sujet de la tradition, sujet détenteur d'une mémoire et de la parole qui lui est assujettie, mais dont la nation de référence, pour le dire avec Fernand Dumont, se trouve dans le passé. Face à ce premier sujet, Perrault caractérise ce qu'il en est du sujet moderne qui court vers l'universel et la reconnaissance, mais qui, dans cette course vers l'avant, se trouve piégé dans l'aliénation, sujet mironnien auquel Perrault luimême s'identifie. Parole sans pays, d'une part, et pays (à faire et en train de se faire) sans parole, d'autre part. Face à cette dichotomie, Perrault semble opérer un double mouvement politique:

1. en filmant les gens de l'île-aux-Coudres, il cherche d'abord à désaliéner le sujet québécois en le remettant en contact avec une parole qui ne s'est pas encore désarrimée de la tradition;

2. il souhaite ensuite prolonger cette mémoire grâce à ces technologies nouvelles d'enregistrement qui sont pour Perrault proprement salvatrices, à condition d'en faire un usage qu'il qualifie luimême à certaines reprises d'iconoclaste:

On dit que les nouvelles techniques reproduisent toujours au début les anciennes habitudes. Il ne faut sans doute pas s'étonner de la profusion des idoles. Mais il faudrait finir par se rendre compte peut-être du regard neuf que la caméra nous procure. Pour la première fois dans l'histoire de l'humanité, l'homme possède un outil fabuleux pour prolonger sa mémoire, pour regarder le monde, pour raconter sa propre humanité, sans avoir à invoquer les dieux de l'imaginaire.

(1995: 175-176) 
Johanne Villeneuve a déjà souligné le platonisme paradoxal de Perrault (2003: 73). En effet, s'il dénonce sans nuance fiction et imaginaire, il ne se prive pas de défendre la capacité d'archivage de la caméra, dont la mémoire technique est mise au service d'une tradition à sauver de l'oubli. Serait-ce que cette parole de la tradition ne parvient plus à se défendre par elle-même? On voit tout ce qu'il y a de problématique à opposer ainsi la mémoire et l'image, d'une part, et surtout la mémoire antiquaire de l'archive au mode d'articulation du passé et du présent propre à la tradition, d'autre part. D'autant que la caméra, dans ce cas, archive non seulement une parole, mais également des gestes et des postures: gestes de tracer la pêche, gestes du conteur (Louis Harvey), immobilité des corps de ceux pour qui la durée indéfinie de la tradition donne le temps de voir venir (Abel Harvey, Alexis Tremblay). Or, il est intéressant de remarquer que le dispositif de la caméra n'affecte pas les corps de la même façon, dans ce premier chef-d'œuvre de la caméra participante qu'est Pour la suite du monde (1963) ${ }^{4}$. Pour un Alexis Tremblay assuré de son savoir et de sa posture, combien de Léopold (fils du précédent) engoncés dans un corps qui ne leur appartient déjà plus? Sujet du passage vers la modernité, à ce titre responsable du projet paradoxal de renaissance d'une pêche abandonnée depuis quarante ans pour les fins $d u$ cinéma, Léopold a les gestes de celui qui, se sachant regardé, perd le contrôle de sa motricité. Dans un ouvrage récent, Étienne Beaulieu écrit que, dans le cinéma de Perrault, «s'énonce cette confiance en une stabilité du passé, en une possibilité de suivre ses traces et, plus encore, de les relever, d'en découvrir la survivance dans le présent» (2007: 116). Mais n'est-ce pas précisément dans cette volonté que réside l'aporie fondamentale de Pour la suite du monde? La survivance du passé qu'elle imprime sur image est avant tout une tentative de se réapproprier des gestes qui sont déjà perdus, sauf pour quelques mémoires sur le point de s'éteindre. Ces images présentent donc le corps de la tradition à préserver, même s'il est déjà en train de se perdre. Le filmer, par conséquent, ce n'est pas seulement lui donner une chance de survie, c'est aussi participer à sa désarticulation. C'est pourquoi ce film, par-delà sa signification directement politique si on le reçoit dans le contexte de l'histoire culturelle du Québec, a pu rejoindre le panthéon du cinéma mondial, puisqu'il est la parfaite illustration d'une perte de l'expérience analysée par Benjamin qui, de par son retard causé par sa position insulaire, se voit «directement» captée par la caméra qui témoigne de son avoir-lieu tout en cherchant à sauver quelques gestes du naufrage de l'oubli ${ }^{5}$.

Filmer les gens de l'île vise donc à mettre en rapport à la fois les auteurs (Perrault, Brault, Carrière, etc.) et les spectateurs avec une parole et des gestes d'ores et déjà perdus. Pourtant, il n'y a qu'à voir Pour la suite $d u$ monde (c'est déjà beaucoup moins vrai pour les autres volets de la "trilogie de l'Île-aux-Coudres») pour sentir qu'il y a quelque chose de plus, une sorte de reste qui appartient à l'archive. C'est ce reste qui en définitive donne toute sa modernité à ce chef-d'œuvre du direct et qui lui fournit sa structure d'événement, soit la rencontre du réel du personnage et du cinéaste dans un échange réciproque qui les affecte de telle sorte que le moindre des énoncés qui en est produit acquiert une portée immédiatement collective. C'est ce que Deleuze, dans L'Image-temps, appelle la politique des «intercesseurs", "c'est-à-dire [le fait] de prendre des personnages réels et non fictifs, mais en les mettant eux-mêmes en état de "fictionner", de "légender" (1985: 289). C'est cette structure de l'événement cinématographique qui a sans doute intéressé Deleuze dans le cinéma de Perrault, cette coïncidence du passé et du présent à l'intérieur d'une parole qui permet d'entrouvrir l'image à la dimension du devenir: devenir-peuple de Perrault lui-même qui, en enregistrant une parole sur le point de se perdre, vise à la production d'une nouvelle énonciation, celle précisément dont il se dépêchait de faire usage dans sa poésie; mais aussi devenir-passé de l'image quant à son statut d'archive, son "avoir-été». Le présent de l'image s'avère ainsi surchargé, pris entre le projet du devenir et la nécessité de l'archive qui répond à la perte irrémédiable de la tradition qu'elle recueille. C'est cette surcharge de l'image-temps qui rapproche Perrault du questionnement - partout répandu dans le roman québécois qui lui est contemporain - de son historicité et de sa métaphore privilégiée, le temps en 
arrêt $^{6}$, et qui pourtant devra recourir à l'arrêt sur image de la photographie pour accéder à sa pleine efficacité.

\section{OMBRE ET LUMIÈRE DU SUJET À VENIR}

Avant de passer à l'analyse de cette historicité propre aux photographies du Nord, il faut s'astreindre au détour qui a permis à Perrault de saisir l'absence comme nouveau corps politique à venir. Ce détour, c'est celui de la métaphore et de l'animal qui lui tient lieu de véhicule, dialectique primordiale qui sert au cinéaste à représenter cette saturation des trois temps de l'image. Le marsouin faisait certes déjà l'objet d'un investissement libidinal déterminant. Mais, en tant qu'objet passéiste d'une pêche depuis longtemps abandonnée, il était surtout le support d'une attente qui concernait un retour du passé. Dans un film dont le titre parfaitement ironique est ainsi renvoyé à une sorte de futur antérieur, Un royaume vous attend 7 , Hauris Lalancette s'avance, fasciné, vers une "taure» en train de meugler, le cri de l'homme et de l'animal devenant ainsi interchangeable afin de dire le désespoir d'un messianisme qui tourne mal ${ }^{8}$. Dans La Bête lumineuse (1982), cette partie de chasse qui tourne au huis clos, c'est plutôt l'absence de l'animal, attendu en vain, qui conditionne la métamorphose de l'homme en proie pour une meute décidée à mener la chasse à terme. On s'en souvient, ce parfait exemple du "cinéma vécu » de Perrault vise surtout à montrer les rites d'un groupe d'amis d'enfance se retrouvant annuellement à Maniwaki pour la chasse à l'orignal. Or, de plus en plus fébrile face à une bête qui se fait attendre, la meute de prédateurs se tournera plutôt, lors de soirées copieusement arrosées, vers celui qui refuse d'abdiquer sa différence, le poète StéphaneAlbert Boulais qui ira jusqu'à déclamer son amitié envers le cuisinier du groupe, Bernard, sous la forme d'un poème ambigu. C'est à partir de cette scène que Gilles Thérien a construit son analyse du film:

En l'absence de l'orignal, [l'arc du poète-chasseur Stéphane-Albert Boulais] devient de plus en plus inutile, et les poèmes s'érotisent. Apollon se transforme en Éros. [...] Bernard réagit comme une vierge farouche à ce qu'il sent poindre. Et, comme Artémis, furieuse d'être surprise au bain par le chasseur Actéon qu'elle transforme en cerf que ses propres chiens dévorent, Bernard va transformer Stéphane-

Albert en proie qu'il offre au reste du groupe. (1999: 171)

Une telle interprétation est particulièrement séduisante, et permet certes de donner sens à l'acharnement dont est victime Boulais. Mais on peut se demander s'il s'agit vraiment du dernier mot de La Bête lumineuse. C'est oublier la véritable leçon d'anthropologie qui est servie au poète par Bernard, le «simple chasseur» et cuisinier de la bande, à la toute fin du périple. Loin d'être apaisé par le sacrifice du bouc émissaire, l'ordre est au contraire toujours aux prises avec une tension que n'a pu relâcher un rituel aux règles transgressées. L'erreur de StéphaneAlbert aura ainsi été de garder pour lui seul une place destinée à se voir occupée à tour de rôle, chacun ayant le droit de se confronter une fois l'an à sa propre mort. Dans ce film, le guet du chasseur est donc une figure de l'attente avant tout factice, puisqu'elle ne sert qu'à pouvoir prendre la place d'une bête qui ne vient pas. Viendrait-elle que le rituel prendrait un tout autre visage.

La bête est donc, dans un premier temps, non pas l'objet de la photographie mais bien un cadre, une figure appelée à céder sa place à l'homme qui se confond avec elle. Et si elle est alors l'objet d'un syncrétisme (entremêlant rites sacrificiels et figuration d'une attente messianique), son apparition dans le champ de la caméra va coïncider, dans L'Oumigmag, avec le retour du commentaire en voix-off qui va venir, si cela était nécessaire, confirmer la fonction métaphorique qui lui est prêtée, le film devenant poème sur image. Du corps de l'homme, offert à la voracité de la meute, au bouf musqué traqué par le regard de la caméra, nouveau «détour par la bête lumineuse, comme l'écrit Perrault, pour comparaître à mes propres yeux» (1995: 114), l'animal devient progressivement la métaphore privilégiée d'une tentative de figuration du dehors, c'est-à-dire de tout ce qui se trouve en périphérie de l'histoire. Et c'est précisément de par cette posture d'extériorité qu'il incarne que l'animal s'impose comme figure substitut d'un corps politique manquant, simple "vivant» dispersé dans un territoire immense et des conditions difficiles. Perrault, d'ailleurs, ne manque pas de livrer 
aux lecteurs les diverses significations qu'il a prêtées à une telle figure:

En vérité le bœuf musqué m'obsède, depuis longtemps, comme une incomparable métaphore [...] d'un peuple oublié en terre d'Amérique par l'histoire et par les Princes. (1995: 32)

On le voit, deux registres, politique et esthétique, s'entremêlent dans la métaphoricité du bøuf musqué. Deux registres qui ne s'excluent pas nécessairement, mais à tout le moins qui ne se situent pas du tout dans la même histoire. En effet, on a vu plus tôt comment L'Oumigmag est un "film sur l'action de filmer", pratique réflexive sur l'objectif documentaire qui fait du bøuf musqué et de sa méfiance la figure de la résistance subjective face au dispositif de la caméra. Dans ces films-bilans sur une pratique continue du documentaire, Perrault est remarquable de clarté et de lucidité quant à la possibilité de capter un réel qui se refuse: l'échec de la rencontre avec le bœuf musqué au moyen d'une «caméra participante», dans L'Oumigmag, fait place dans Cornouailles au retour du téléobjectif. Mais, ce qui étonne, c'est le retour du discours de la survivance, reçu en tant que tel sans être remis en question par certains critiques (Marie, 1999: 136). C'est peut-être qu'au même titre que le bœuf musqué se refuse à la caméra, la métaphore dont on l'affuble refuse d'y adhérer parfaitement. En effet, que signifie donc faire du bøuf musqué une métaphore du sujet québécois toujours perçu dans une posture de repli? C'est avant tout ajouter à l'image la dimension du temps. Car, au-delà du contenu discursif propre à la survivance, ce qu'il faut rappeler, c'est que cette posture était fondamentalement une inscription formelle dans l'histoire, manière de se préserver une place dans ce qui était encore à venir. Cherchant dans le bœuf musqué l'aura d'ancestralité qui vise à prendre l'histoire à rebours, Perrault peut ainsi, le temps de son documentaire, se servir de cette «bête qui précède l'histoire [pour] recommencer l'Amérique. Mettre au monde un pays plus ancien que l'homme» (1995: 62 et 157). C'est donc à même cette figure qu'il cherche à cerner cette historicité québécoise constituée d'une intrication particulièrement complexe des dimensions de la temporalité, puisque l'origine, ou disons le commencement, sans cesse reporté, est à situer dans une projection constante vers l'avant. Dès lors, monter vers le Nord signifiera surtout remonter le temps, voire en ralentir l'écoulement.

Ce qui me semble fondamental, dans L'Oumigmag, c'est donc le problème de l'instant, cette "vitesse folle» par laquelle «l'instant présent» retombe immédiatement dans le passé (ibid.: 25). Aussi Perrault s'adjoint-il une autre métaphore nécessaire à la saisie du corps à venir dans le cadre de l'image. Métaphore directement temporelle qui préside à cette appréhension du Nord, le «soleil de minuit» perceptible au-delà du cercle polaire suspend la durée du jour et ruine le calendrier. Ce n'est que dans sa lumière paradoxale que Perrault pourra se dire «enfin libéré du temps. Ou encore menacé par le néant, cet ultime passage vers un au-delà où le temps s'inverse, s'engouffre et s'anéantit» (ibid.: 51; je souligne). Ainsi, à l'image saturée de temps du début de l'œuvre, prise entre un passé en train de se perdre, à préserver, et un avenir à désobstruer en renouant avec sa propre mémoire, le prétexte d'une bête métaphorique à filmer pour se signifier sa propre distance à l'égard du monde débouche soudain sur un nouveau devenir de l'image, celui de filmer le jour le plus long. C'est ce temps quasi immobile qu'il cherchera à capter par le biais de la photographie dans Le Mal du Nord: «Comme si le temps s'arrêtait là, ou le soleil. On se prend alors pour Jéricho» (Perrault, 1999: 196). Temps parfaitement messianique, on le voit, ne serait-ce que par le rappel de cette figure biblique de Jéricho 9 .

\section{L'INSTANT PHOTOGRAPHIQUE DU SOLEIL DE MINUIT}

Le Mal du Nord, ce récit d'un bref voyage vers le pôle, est aussi bien l'abandon de l'animal comme ultime incarnation du sujet politique québécois que le deuil d'un dernier film que Perrault n'aura pas tourné, et «qui raconterait la vie d'un iceberg depuis son vêlage jusqu'à sa sublimation dans les eaux chaudes des grands bancs" (ibid.: 260). C'est cette fois la glace - glace de mer et morceaux de banquise à la dérive - qui sera son objet privilégié. Mais avant d'amorcer l'analyse de quelques clichés, il importe d'expliciter le titre du récit, dans la mesure 
où il implique toute cette dimension du désir qui fait de ces photos de véritables objets mélancoliques. En effet, qu'est-ce donc que ce mal du Nord? Tout le contraire d'un mal de mer, malgré la perte des repères que la blancheur infinie provoque, qui sera bien plutôt vécue sur un mode proprement extatique. Ce mal du Nord, expression que Perrault emprunte au peintre René Richard, qui y a vécu en solitaire de longues années et en a ramené ses couleurs, est plutôt forgé sur le lieu commun du "mal du pays", cet objet perdu qui, parce qu'il est perdu, est infiniment désiré, voire désiré de manière projective parce que l'on sait qu'il sera sous peu perdu (Agamben, 1998: 48). Il s'agit donc une fois de plus d'une dialectique qui met aux prises le désir et le temps, voire un désir de l'histoire qui assume une fois pour toutes une mélancolie dont l'absence de tout corps, humain ou animal, marque le signe le plus sûr de l'insuffisance du sujet politique.

Ainsi, la glace photographiée par Perrault se définit par au moins deux caractéristiques: son statut éphémère et la multiplicité des formes et des couleurs. Il racontera ainsi comment le Nord est proprement «indescriptible», et que la plus grande déception pour soi et pour les autres, au retour, réside dans l'incapacité de rendre compte de sa beauté paradoxale: «Même la photo, écrit-il, n'arrive pas à rendre justice à ce délire de couleurs et de formes. Sans figuration" (1999: 195). Or ce délire de formes et de couleurs n'est pas sans conséquence sur le geste du photographe, lui qui ajoute aussitôt comment tout, sans cesse, est à «recommencer» (ibid.), dans un jeu de perpétuelle refondation qui retient déjà le cinéaste depuis La Grande Allure (1985). Tout au plus évoquera-t-il les métaphores de la dentelle, des tissures et des textures, ce qui du même coup fait de la glace une métaphore à la fois de l'écriture et du montage, ce jeu de texture à priser et repriser. Telle photographie, retenue pour ses "exubérances de formes» et ses «intensités de couleurs», sera par exemple décrite en tant que "beauté sans signification" (Perrault, 1999: 325). Photographier la glace de mer s'avère donc pour Perrault une façon d'ouvrir l'image à une forme d'abstraction qui connote l'absence qui en vient ainsi à saturer l'espace photographique 10.
Il y a donc deux objets bien distincts au sein de ces photographies du Nord. D'abord la glace de mer aux formes enchevêtrées qui ouvre les frontières du cadre, ce qui fait du Nord un non-lieu, antichambre sans forme d'où il serait loisible d'imaginer le politique plus que jamais à venir. Vient ensuite l'objet privilégié du Mal du Nord, l'iceberg, objet d'ailleurs davantage désiré que photographié, objet raconté, cerné par une parole qui le magnifie au point d'en faire un véritable fragment d'infini aux prises avec le temps (que Michel Garneau désignera dans La Grande Allure d'un nom que Perrault se rappellera: la «molaire de Dieu "). Un objet qui vise à figurer non plus le sujet, mais bien son historicité paradoxale, telle qu'elle a pu être évoquée par Michel Serres en conclusion de La Grande Allure 11, c'est-à-dire la contraction d'une temporalité démesurée pourtant sur le point de disparaittre. En effet, qu'est-ce au juste qu'un iceberg? C'est un immense morceau de glace millénaire, mis bas, selon une métaphore de parturition fréquemment utilisée par Perrault, par un glacier dans la mer. À cet égard, le statut phénoménologique de la photographie avancé par Roland Barthes semble particulièrement approprié pour aborder un tel objet. Car l'iceberg, sous l'objectif de Perrault, est plus que jamais frappé par la mort: «ayant-été» millénaire qui, au moment où il se retrouve détaché de sa matrice, est appelé à fondre dans un délai de deux à trois ans. Regardant ce cliché, nous sommes donc, il va de soi, persuadés de sa disparition: il a disparu, il va disparaitre (Barthes, 1980: 124 et 150). Je rappelle cette évidence pour bien mettre en lumière la fonction du Nord dans cette photographie qui, chez Perrault, se confond avec le leitmotiv du soleil de minuit, ce temps suspendu où le soleil semble obéir à l'injonction de Josué. C'est alors, il me semble, que l'iceberg accède à une métaphoricité qui le fait rejoindre les trois objets de l'image cinématographique chez Perrault (après le corps de la tradition, la proie désirée de la bête lumineuse et le corps métaphorique de l'oumigmag). Comme si Perrault ne pouvait impressionner la pellicule de cet objet déjà perdu qu'à le faire à la lumière de ce soleil de minuit qui en suspend la course: "comme si le temps s'arrêtait d'un seul coup. Sur le coup de minuit. On n'avance plus dans le calendrier» (Perrault, 1999: 225). 
Ce temps suspendu lui permet alors de décrire ce «quelque chose qui transcende la mince pellicule de ce que nous appelons l'histoire" dans ces «masses d'inertie polaire» (ibid.). Cette image d'une pellicule à transcender, voire à perforer, est particulièrement éloquente si l'on se souvient, avec Hubert Damisch, que «le même siècle qui a inventé la photographie a inventé l'histoire, cette hystérique [...] qui n'existe que si on la regarde" (2001: 21). Prendre un iceberg comme objet est donc pour Perrault une manière de mettre la photographie et l'histoire en échec, c'est-àdire neutraliser l'écoulement temporel au fondement de l'un et l'autre dispositifs pour ouvrir une toute nouvelle historicité. Si on peut définir, avec Kant, un acte "comme un point où "l'éternité intervient dans le temps" [...] geste "hors temps" et "éternel” de dépassement de l'éternité qui simultanément ouvre la dimension de la temporalité et de l'historicité" (Žižek, 2008: 136-137), alors le choix de cet objet et surtout de la lumière paradoxale du soleil de minuit pour le photographier est précisément l'acte au moyen duquel Perrault vise à faire coïncider le corps politique manquant et l'expérience temporelle singulière de l'historicité québécoise. Aussi, après le marsouin de l'Île-aux-Coudres, objet archaïque d'une chasse depuis longtemps abandonnée, l'orignal manquant de La Bête lumineuse et le bœuf musqué qui demeure énigmatique, c'est-à-dire inapprochable, l'iceberg s'avère-t-il donc l'ultime figure de l'œuvre chargée d'épouser un imaginaire de la perte. Et sans doute ne pouvait-il qu'être photographique pour bien marquer sa nature transitoire immortalisée par le temps qui au Nord suspend sa course.

Nous serons ainsi passés du corps politique préservé de l'ancêtre insulaire, présenté en tant qu'idéal, à une image où, dans l'intervalle, le corps s'est absenté, aussi bien dire d'un imaginaire à un autre. La photographie, parallèle en cela à l'évolution du cinéma, sert à Perrault à témoigner d'une absence: en évinçant progressivement le corps du cadre de l'image, il signale par le fait même l'absence proprement politique d'une subjectivité: corps-sujet dont la parole, pour avoir existé dans l'espace de la tradition, peu à peu disparaît dans un fade out dont il a cherché à recueillir la trace. Photographier les icebergs, objets transitoires par excellence, c'est donc tourner son objectif vers une absence qui n'en est pas moins une forme de réel, celle d'un objet marqué par le temps qui n'en finit plus de ne pas accéder à l'histoire, malgré les tentatives d'en immobiliser la course, ou la perte, par le geste de Josué pour lui donner le temps nécessaire.

\section{NOTES}

1. Voir en particulier le tout petit texte d'Agamben intitulé Qu'est-ce qu'un dispositif? (2007), dans lequel il explicite sa filiation avec Foucault, auteur de la notion, tout en la généralisant pour en faire l'une des deux "classes " de son ontologie avec le "vivant" et le sujet comme "tiers", produit du corps à corps entre les deux. Il convient donc de noter comment le dispositif, à la fois chez Foucault et Agamben, est avant tout une "machine qui produit des subjectivations, [ce] par quoi il est aussi une machine de gouvernement" (Agamben, 2007: 42).

2. Voir les «notes" que Perrault a fait parvenir à Michel Marie à la lecture d'une version préliminaire de son article «À propos de L'Oumigmag et de Cornouailles", en particulier la note 7 (1999: 145).

3. On trouve un bon exemple de cette double dimension dans un commentaire de Michel Brault, coréalisateur et grand artisan de Pour la suite $d u$ monde, qui raconte, dans le documentaire qui lui est consacré dans le coffret regroupant ses œuvres et lancé par l'ONF en 2005, comment Grand-Louis, l'un des personnages les plus mémorables, «était [toujours] meilleur quand la caméra tournait». Il soulignait ainsi la grande question soulevée par les objectifs du direct: celle de l'effet de la caméra sur les sujets filmés, c'est-à-dire la "fiction" qu'implique la seule présence d'une technologie d'enregistrement auprès des sujets, ce pouvoir de la technologie de les faire "légender». 
4. On sait que cette technique est surtout le fait de Michel Brault, technique qui repose sur l'utilisation du grand angulaire dont le premier essai, Les Racquetteurs, a fait de Brault le chef opérateur dominant des années 1960 suite à sa rencontre avec Jean Rouch au séminaire Flaherty.

5. À ce titre, on est frappés de la concordance de cette aporie avec l'une des propositions d'Agamben dans ses « Notes sur le geste", proposition qui résulte de la mise en relation de la perte de l'expérience analysée par Benjamin dans «Le narrateur», des Études cliniques et physiologiques sur la marche de Gilles de La Tourette (celuilà même qui venait, un an plus tôt [1886], de décrire son fameux syndrome qu'Agamben qualifie de "catastrophe généralisée de la sphère de la gestualité») et de la décomposition du mouvement de Muybridge effectué à l'aide de la photographie: «Dans le cinéma, une société qui a perdu ses gestes cherche à se réapproprier ce qu'elle a perdu, et en consigne en même temps la perte" (Agamben, 1995: 63). 6. Sur la dimension messianique, chez Victor-Lévy Beaulieu, d'un tel temps en arrêt, c'est-à-dire l'annonce d'un événement transcendant l'histoire indéfiniment reporté à un au-delà toujours à venir, je me permets de renvoyer à mon article (2005: 109-110).

7. Le premier volet du "cycle abitibien" dans lequel Perrault revient sur cette utopie de la colonisation du Nord qui, du curé Labelle à l'abbé Maurice Proulx, pionnier du documentaire, a animé les élites canadiennes françaises jusqu'à l'aube de la Révolution tranquille.

8. Voir, sur cette question, Johanne Villeneuve (2003: 76).

9. Perrault confond en fait la ville de Jéricho avec la figure de Josué, qui arrêta le soleil le temps de compléter une bataille victorieuse, dans la reconquête de la terre de Canaan. Si Jéricho est la première ville conquise, le geste de Josué a lieu lors de la bataille de Gabaon. 10. Ces photographies, à l'égal de certains tableaux nordiques de Borduas qui apparaissent comme autant d'intertextes, sont à situer au carrefour des cinquième et sixième axes de représentations du Nord, tels que définis par Daniel Chartier dans sa typologie de l'imaginaire du Nord, soit la nordicité vécue en tant qu' "épreuves", physiques ou initiatiques, et le Nord esthétique défini selon un lexique de la pureté et de l'éternité, voire en tant qu'opposition structurante de noirceur et de blancheur (Chartier, 2004 : 18-19).

11. On se souvient peut-être du mot du philosophe, que Perrault, au moyen du montage, articule sous la forme d'une réponse à Michel Garneau, qui déplorait l'inexistence de "son " fleuve fait d'affirmation et d'appartenance, réponse d'autant plus frappante qu'elle constitue les derniers mots du film: «Peut-être que vous êtes trop pressés. C'est vrai que vous n'en êtes pas revenus d'être arrivés là. Mais quand on arrive dans un pays, combien faut-il de temps pour l'habiter? Peut-être faut-il des millénaires ».

\section{RÉFÉRENCES BIBLIOGRAPHIQUES}

Agamben, G. [1995]: Moyens sans fins, Paris, Rivages;

[1998]: Stanze. Parole et fantasme dans la culture occidentale, Paris, Rivages;

[2005]: Profanations, Paris, Rivages;

[2007]: Qu'est-ce qu'un dispositif?, Paris, Rivages.

BARTHES, R. [1980]: La Chambre claire. Note sur la photographie, Paris, Gallimard/Seuil.

BEAULIEU, É. [2007]: Sang et Lumière. La communauté du sacré dans le cinéma québécois, Québec, L’instant même.

Chartier, D. [2004]: «Au Nord et au large. Représentation du Nord et formes narratives", dans J. Bouchard, D. Chartier et A. Nadeau

(dir.), Problématiques de l'imaginaire du Nord en littérature, cinéma et arts visuels, Montréal, Figura, no 9, 18-19.

DAmISCH, H. [2001]: La Dénivelée. À l'épreuve de la photographie, Paris, Seuil.

Deleuze, G. [1985]: Cinéma 2. L'Image-temps, Paris, Minuit.

FOUCAULT, M. [1975]: Surveiller et punir. Naissance de la prison, Paris, Gallimard.

Hartog, F. [2003]: Régimes d'historicité. Présentisme et expériences du temps, Paris, Seuil.

INKEL, S. [2005]: «Le temps suspendu. Messianisme, arrêt de l'histoire et politique du Livre chez Victor-Lévy Beaulieu ", Voix et Images, vol. XXX, n² 2 (89), hiver, 107-123.

LACROIX, Y. [2006]: «Fragments d'un bestiaire", dans S.-A. Boulais (dir.), Le Cinéma au Québec. Tradition et modernité, Montréal, Fides.

MARIE, M. [1999]: "À propos de L'Oumigmag et de Cornouailles", dans P. Warren, P. (dir.), 133-146.

Perrault, P. [1995] : L'Oumigmatique ou l'objectif documentaire, Montréal, L'Hexagone; - [1999]: Le Mal du Nord, Hull, Vents d'Ouest.

THÉRIEN, G. [1999]: «Bête lumineuse », dans P. Warren (dir.), 163-175. VIlleneuve, J. [2003]: « Pierre Perrault, poète et cinéaste», L'Esprit créateur, vol. XLIII, n² 2, 72-80.

WARREN, P. (dir.) [1999] : Pierre Perrault, cinéaste-poète, Montréal, L'Hexagone.

ŽIžEK, S. [2008] : Fragile absolu, Paris, Flammarion.

\section{Films de Pierre Perrault}

Pour la suite du monde, coréal. M. Brault, 1963, 105 min. Un royaume vous attend, coréal. B. Gosselin, 1975, $110 \mathrm{~min}$. La Bête lumineuse, 1982, $127 \mathrm{~min}$.

La Grande Allure (2e partie), 1985, $73 \mathrm{~min}$.

L'Oumigmag ou l'objectif documentaire, 1993, 28 min.

Cornouailles, 1994, $52 \mathrm{~min}$. 